\title{
Was ist wichtiger als eine Gehaltserhöhung?
}

Der „War for Talents“ und die Digitalisierung der Bildung gelten derzeit als Megatrends in der Diskussion um Recruitment und Fort- und Weiterbildung. Denn viele Mitarbeiter sehen die Weiterbildung als Fundament für ihren beruflichen Erfolg und schauen, welche Angebote und Rahmenbedingungen ihnen die Unternehmen bieten.

Die von der PFH Private Hochschule Göttingen durchgeführte Studie „Digitalisierung in Bildungsprozessen“ hat deshalb untersucht, welche Instrumente bei der Mitarbeiterförderung im deutschen Mittelstand eingesetzt werden. Die Autoren der Studie, Professor Bernt R. A. Sierke, Joachim Algermissen und Stefan Brinkhoff, haben zudem insbesondere den Stand der Digitalisierung in den Bildungsprozessen im Mittelstand ermittelt. Befragt wurden 5.338 Geschäftsführer und CEOs deutscher mittelständischer Unternehmen.

Wichtigste Befunde: Insgesamt gibt jedes zweite Unternehmen Probleme bei der Rekrutierung von Mitarbeitern an, insbesondere im Bereich der Fachkräfte. Den War for Talents nehmen überraschenderweise aber erst 38 Prozent von ihnen wahr.

\section{Mittel der Wahl: Förderung per Studium}

Nahezu alle Unternehmen (94 Prozent) setzen Fort- und Weiterbildungen zur Mitarbeiterförderung ein. Diese weisen damit sogar einen höheren Stellenwert als Gehaltserhöhungen auf. Mehr als jedes zweite Unternehmen bietet seinen Mitarbeitern berufsbegleitende Studienprogramme zur Fort- und Weiterbildung an. Duale Studiengänge sehen 79 Prozent der Befragten dabei als sinnvolles Instrument für die Bewältigung des Fachkräftemangels an, insbesondere, weil sie als praxisnäher gelten als nicht-duale Programme. Rund 85 Prozent der befragten Unternehmen würden ihre Mitarbeiter finanziell bei einem solchen Studium unterstützen.

Rund jedes zweite deutsche mittelständische Unternehmen kooperiert bereits mit einer Hochschule, die berufsbegleitende Studienprogramme anbietet. Der Schwerpunkt dieser Kooperationen liegt auf (Fach-)Hochschulen; Berufsakademien liegen deutlich abgeschlagen dahinter. Klassische Universitäten spielen bei berufsbegleitenden Programmen eine eher untergeordnete Rolle.

\section{Digitalisierung von Studienangeboten}

Rund 75 Prozent der befragten Unternehmen nehmen zudem Digitalisierung als wichtiges Thema wahr. In diesem Rahmen

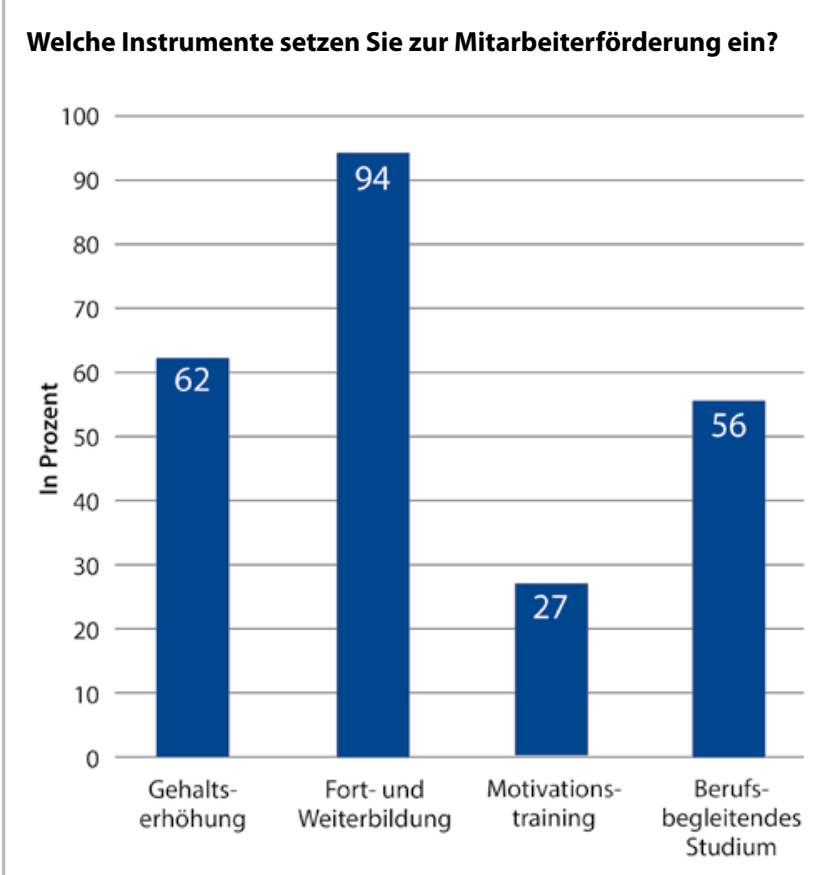

Quelle: Sierke, B. R. A./Algermissen, J./ Brinkhoff S. (2016): Digitalisierung in Bildungsprozessen. Eine Studie im deutschen Mittelstand, Göttingen.

wird dem E-Learning von mehr als jedem zweiten Unternehmen bei der Aus- und Weiterbildung seiner Mitarbeiter ein hoher Stellenwert beigemessen. Knapp 60 Prozent der befragten Unternehmen könnten sich duale Studiengänge als Fernstudium - und hier insbesondere mit digitalisierten Inhalten - vorstellen. Zwar haben bisher nur etwas weniger als die Hälfte der deutschen mittelständischen Unternehmen Erfahrungen mit Fernstudiengängen gemacht. Diejenigen Unternehmen, die sich dafür entschieden haben, geben dafür aber fast ausschließlich positive Rückmeldungen.

„Eigentlich teilen uns die mittelständischen Unternehmen in diesen Studienergebnissen mit, wie für sie die optimale Fördermaßnahme für Mitarbeiter aussehen soll: Fernstudiengänge, die in erster Linie zeit- und ortsunabhängig für die Mitarbeiter studierbar sind, mit nur wenig Ausfallzeiten im Unternehmen und mit Praxisthemen, die mit den Studieninhalten verknüpfbar sind", so das Fazit zur Studie von Professor Bernt R. A. Sierke, dem federführenden Autor der Untersuchung.

Peter Diehl

Ð Ein E-Book der Vollversion der Studie finden Interessierte unter www.pfh.de/studien. 